Research Article

\title{
Data Reliability Analysis of Wireless Sensor Nodes considering Perturbation
}

\author{
Shukun Jin,, ${ }^{1,2}$ Yawen Xie $\mathbb{D}^{1},{ }^{1}$ Yanzi Gao, ${ }^{3}$ Guohui Zhou $\mathbb{D}^{1},{ }^{1}$ Wei Zhang, ${ }^{1}$ Shuaiwen Tang, \\ and Wei He iD ${ }^{1,4}$ \\ ${ }^{1}$ School of Computer Science and Information Engineering, Harbin Normal University, Harbin 150025, China \\ ${ }^{2}$ Guangdong Business and Technology University, Zhaoqing 526020, China \\ ${ }^{3}$ Institute of Advanced Materials and Technology, University of Science and Technology Beijing, Beijing 10083, China \\ ${ }^{4}$ Rocket Force University of Engineering, Xi'an 710025, China
}

Correspondence should be addressed to Guohui Zhou; zhouguohui@hrbnu.edu.cn

Received 29 January 2021; Revised 14 June 2021; Accepted 17 June 2021; Published 23 July 2021

Academic Editor: Giovanni Diraco

Copyright (c) 2021 Shukun Jin et al. This is an open access article distributed under the Creative Commons Attribution License, which permits unrestricted use, distribution, and reproduction in any medium, provided the original work is properly cited.

Wireless Sensor Network (WSN) is widely used in precision engineering, which requires strict data accuracy. Therefore, it is of practical value to evaluate the reliability of WSN data. Considering the complexity of the real environment, the sensor is bound to be affected by the disturbance factors. Currently, the research result of WSN data disturbance is not ideal. Because the results of reliability analysis are not necessarily credible under a disturbance environment. Thus, it is necessary to judge the reliability of sensor nodes in the disturbance environment. Therefore, disturbance analysis is introduced. In this paper, the temporal correlation and spatial correlation of measured data of WSN nodes are taken as reliability indicators. Through the disturbance analysis method to simulate the disturbance in the working process of nodes, a data reliability evaluation model of WSN nodes is proposed, which is based on the evidence reasoning (ER) rule in the disturbance environment. Based on the wireless sensor experiment of the Intel Berkeley research laboratory, the rationality of the model is explained in this paper. ER rule considering disturbance provides an effective method to analyze the reliability of WSN data.

\section{Introduction}

Sensors are mainly used for real-time monitoring of complex monitoring areas, to grasp the attribute data of the regional environment. They play an essential role in the environment [1], food [2], industry [3], and other fields. The characteristics of large quantity, wide range, limited energy, complex environment, and network stability are possessed by WSN nodes $[4,5]$. Because of these characteristics, sensors are easily disturbed by environmental factors, network problems, and node failures. The data collected by sensors is not completely reliable, which may cause incalculable losses in engineering practice or combat tasks. Therefore, it is of great significance to analyze sensor nodes' data reliability and adaptability under disturbance conditions.
To solve the problem of WSN data reliability, many scholars have given different research methods. From the perspective of sensor failure analysis, Gao et al. [6] proposed a new distributed and adaptive trust measurement method for MANET. Direct trust is calculated through inter-node communication, energy, and recommendation methods in this method. In addition, the propagation distance of the trust degree is considered to calculate the indirect trust degree, which greatly improves the ability to distinguish malicious nodes. He et al. [7] proposed a reliability evaluation method based on a hierarchical BRB (Belief Bule Base), which analyzed WSN reliability evaluation from internal failures and external attacks; Peng et al. [8] proposed a detection method based on sensor network time series data, which uses the degree of difference between the calculated test data and the normal 
interval to determine the source of the fault; In the above method, the aspects of detecting malicious nodes and sensor failures are analyzed. These methods have better analysis results when the data changes drastically. However, for insignificant data fluctuations, satisfactory results cannot be achieved.

From the perspective of improving the reliability of information transmission, Zhang et al. [9] proposed an energybalanced routing method based on forwarding perception factors. In this method, the selection of the transmission node is carried out by analyzing the weight of the transmission path and the forward energy density. By studying the method of selecting cluster heads in traditional clustering algorithms, it is easy to cause problems of poor network stability and reliability. Ge et al. [10] proposed a new passive multi-hop clustering algorithm. Zhang et al. [11] proposed an effective data clustering method based on compressed sensing by analyzing the advantages of compressed sensing technology in wireless sensor network data aggregation. Zhang et al. [12] proposed a new method of tensor heterogeneous integration learning data loss estimation based on fuzzy neural networks to solve the situation of data loss and abnormality. Alipio et al. [13] proposed a reliable transmission protocol with RT-CaCC cache-aware congestion control mechanism, using cache management strategies to alleviate data packet loss in the network. Sharma D et al. [14] discussed the influence and interdependence of different node heterogeneity on routing decisions. They improved the reliability of WSN in various scenarios. In the above method, the reliability of the sensor is improved from aspects such as optimizing the data transmission strategy. And these methods reduce the loss or distortion of data in the process of transmission. However, from the perspective of sensor data change, the effect is not satisfactory.

The network partition is divided by the distance from the node to the base station. From the perspective of optimizing network routing protocols, Liu et al. [15] proposed a new unequal cluster routing protocol that considers energy balance. Zhang et al. [16] proposed a new routing protocol QG-OLSR by improving the quantum genetic strategy and combining optimized link-state routing. After that, considering the two properties of frequent network topology replacement and poor communication link reliability in the vehicle ad hoc network, a new adaptive routing service algorithm was proposed [17]. The shortcomings of dynamic source routing protocol in mobile ad hoc networks are analyzed and optimized. Liu et al. [18] proposed a new dynamic source routing protocol based on genetic algorithm-bacterial foraging optimization. During mobile edge computing, node movement and node energy caused link failures, and network delays caused by failures were discovered. Chen et al. [19] proposed a multi-path routing protocol based on link lifetime and energy consumption prediction. Xiang et al. [20] proposed two new reliability indicators: generalized terminal reliability and average generalized terminal reliability and optimized the design of WSN. The above methods are analyzed from the perspective of optimizing network routing methods. However, the processing of sensor data is lacking.

In the above discussion, the reliability of WSN is mainly focused on various routing protocols, topology design or net- work coverage, fault diagnosis, and connectivity optimization. However, the above research ignores that the main function of WSN is to monitor the target and collect attribute information. And the real-time data reliability evaluation is critical and vulnerable to disturbance. Moreover, WSN realtime data has the characteristics of unsupervised. However, the general unsupervised learning methods are vulnerable to extreme point interference. They cannot adequately consider the impact of disturbance on WSN, such as databased clustering methods and knowledge-based analytic hierarchy process. Therefore, for the reliability evaluation of WSN data, it is necessary to consider multiple reliability characteristics based on WSN data. However, due to environmental disturbance, and the assessment involves qualitative and quantitative information and its uncertainty. The above methods have no good effect on this problem.

D-S evidence theory [21] and ER algorithm [22] are further developed into ER rule [23]. In practical application, the evidence is not completely reliable. Therefore, to make the evidence more in line with the actual situation, the concept of evidence reliability is introduced into ER rule to reflect the reliability of evaluation information [21]. In ER rule, weight and reliability are fully considered. Experts' subjective experience and objective data are combined to describe the data with belief distribution. It has significant advantages in dealing with information uncertainty [24]. And the whole process is interpretable. Data features are extracted to evaluate WSN data's reliability without supervision, which can effectively solve the above problems. Due to their strong handling capacity in terms of uncertainty and the characteristics of considering the weight and reliability of evidence, ER rules are widely used in many fields, such as evaluation and decision-making. For example, Zhao et al. [25] proposed an on-line security evaluation method based on ER. This method fuses the state of "history", "present" and "future" of the system to evaluate the comprehensive security level of the system. Zhou et al. [26] extended ER rule to the MADM problem in a group decision-making environment. The interval weight and reliability of experts and evidence are fully used to evaluate the service life of electric vehicles. In this paper, the measured data of WSN is taken as the research object. And ER rule is applied to the reliability evaluation of WSN data. Considering the disturbance environment's influence on the evaluation process, the method of WSN data reliability evaluation under the disturbance environment is proposed based on ER rule.

The remainder of this paper is organized as follows. In Section 2, a feasibility analysis of WSN data reliability is presented to determine the whole evaluation process. In Section 3 , the working mechanism model of WSN is constructed to describe a series of methods used from the determination of evaluation indicators to the final analysis of evaluation results. The model's effectiveness is validated by experiments and case studies in Section 4. The conclusion is reported at the end of this paper.

\section{Description of the Problem}

WSN is easily affected by disturbance factors because of its operational characteristics. Therefore, disturbance factors 
can cause data anomaly or even loss, such as environmental countermeasures and network failure. Moreover, the evaluation results are not credible in different interference environments. The abnormal monitoring data can reflect the influence of disturbance on sensor nodes. Corresponding, the monitoring data are reflected through the disturbance analysis whether we want to adapt sensor nodes to a particular environment. This provides ideas for the follow-up experiments. Disturbance analysis was first proposed by Ho and applied to discrete event dynamic systems. Through the experimental method, a disturbance sample trajectory is added to the original data to analyze the system performance indicator's sensitivity to a critical parameter [27]. Therefore, this paper simulates the uncertainty from the perspective of monitoring data in the actual environment. The reliability evaluation model of sensor nodes data is established in the disturbed environment based on ER rules. This model is used to quantify the adaptability of sensor nodes to different disturbances, so as to analyze the reliability of sensor data. Combine the problems that may occur in the actual project, and make the following summary:

Problem 1. Construct an evaluation indicator system. Many factors can reflect the reliability of sensor node data. A reasonable evaluation indicator system is the basis of accurate evaluation of node data reliability.

$$
S=\left\{x_{1}, \cdots, x_{i}, \cdots, x_{I}\right\}
$$

where $S$ is the indicator system. $x_{i}$ is the ith indicator. And $I$ is the number of indicators.

Problem 2. The reliability evaluation model of sensor nodes data is constructed based on ER rule under disturbance environment. Based on considering the indicator weight and reliability, all indicator information can be fused by ER rule to evaluate the data reliability of sensor nodes. Besides, the reliability of data collected by sensor nodes is related to the degree of disturbance. To better simulate the actual work scene, this paper proposes the following model:

$$
Y(t)=\Psi[z(t), w, r, \sigma, \Delta z(t)]
$$

where $z(t)$ is the indicator data at time $t . w$ is the weight of the indicator. $r$ is the reliability of the indicator. $\sigma$ is the disturbance intensity. $\Delta z(t)$ is the disturbance variable that the indicator data receives at time $t . \Psi(\bullet)$ is the nonlinear function corresponding to the model and $Y(t)$ is reliability evaluation result under the influence of disturbance at time $t$.

In this paper, the non-disturbance case is taken as a particular case of the above model. In other words, disturbance intensity $\sigma$ and disturbance variables $\Delta z(t)$ are not considered. The following formula is obtained:

$$
y(t)=\Gamma[z(t), w, r]
$$

where $\Gamma(\bullet)$ is the nonlinear function corresponding to the ER rule. $y(t)$ is the reliability evaluation result at time $t$ without disturbance.

Problem 3. The reliability of sensor nodes is analyzed for the disturbed environment. The adaptability of sensor nodes is different for different disturbance environments. If the impact of disturbance on the data exceeds a specific range, that is, the sensor nodes cannot normally work in this environment. And the reliability of sensor data is affected. Therefore, other features of WSN need to be adjusted. The formula can be expressed to quantify the adaptability of sensor nodes to disturbance environment as follows:

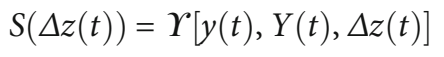

where $S(\Delta z(t))$ represents the disturbance factor of the indicator data at $t$ times affected by the disturbance variable $\Delta z$ $(t)$. It can be used to measure the adaptability of WSN nodes in a disturbed environment.

The whole evaluation process is shown in Figure 1:

\section{Model Implementation Process}

3.1. Determine the Evaluation Indicator System. From the perspective of monitoring data, the reliability of sensor node data is evaluated. The factors reflecting the reliability of node data are determined. And a reasonable evaluation indicator system is constructed. Due to the complexity of the environment and the limitation of node health, there are many indicators that can reflect sensor node data reliability. For example, the values of environmental attributes measured by the same node will not fluctuate significantly in a short time, which is based on the characteristics of WSN. This represents the node's time correlation; since the monitoring environment is similar between different nodes in the neighborhood, the measured environmental attribute values should be similar and have the same changing trend. This represents the spatial correlation of the nodes. In this paper, temporal correlation and spatial correlation are used as the evaluation indicators to evaluate sensor node data [28]. Taking temperature data as an example, the temporal correlation and spatial correlation of temperature data are extracted to evaluate sensor node data reliability.

3.1.1. Time Correlation. In probability statistics, the standard deviation is usually used to measure the degree of statistical distribution, which reflects the degree of dispersion of data within a group. Because the temperature data measured by sensor nodes in a short time should not have the characteristics of large fluctuations. Therefore, the method of calculating the standard deviation of data in a time period is used to reflect the time correlation of the node in this paper:

$$
A_{i}^{m}(t)=\left[a_{i}^{m}(t-n+1), \cdots, a_{i}^{m}(t-1), a_{i}^{m}(t)\right]
$$

Among them, $a_{i}^{m}(t)$ represents the raw data of node $m$ about indicator $x_{i}$ at time $t . A_{i}^{m}(t)$ represents the $n$-Dimen 


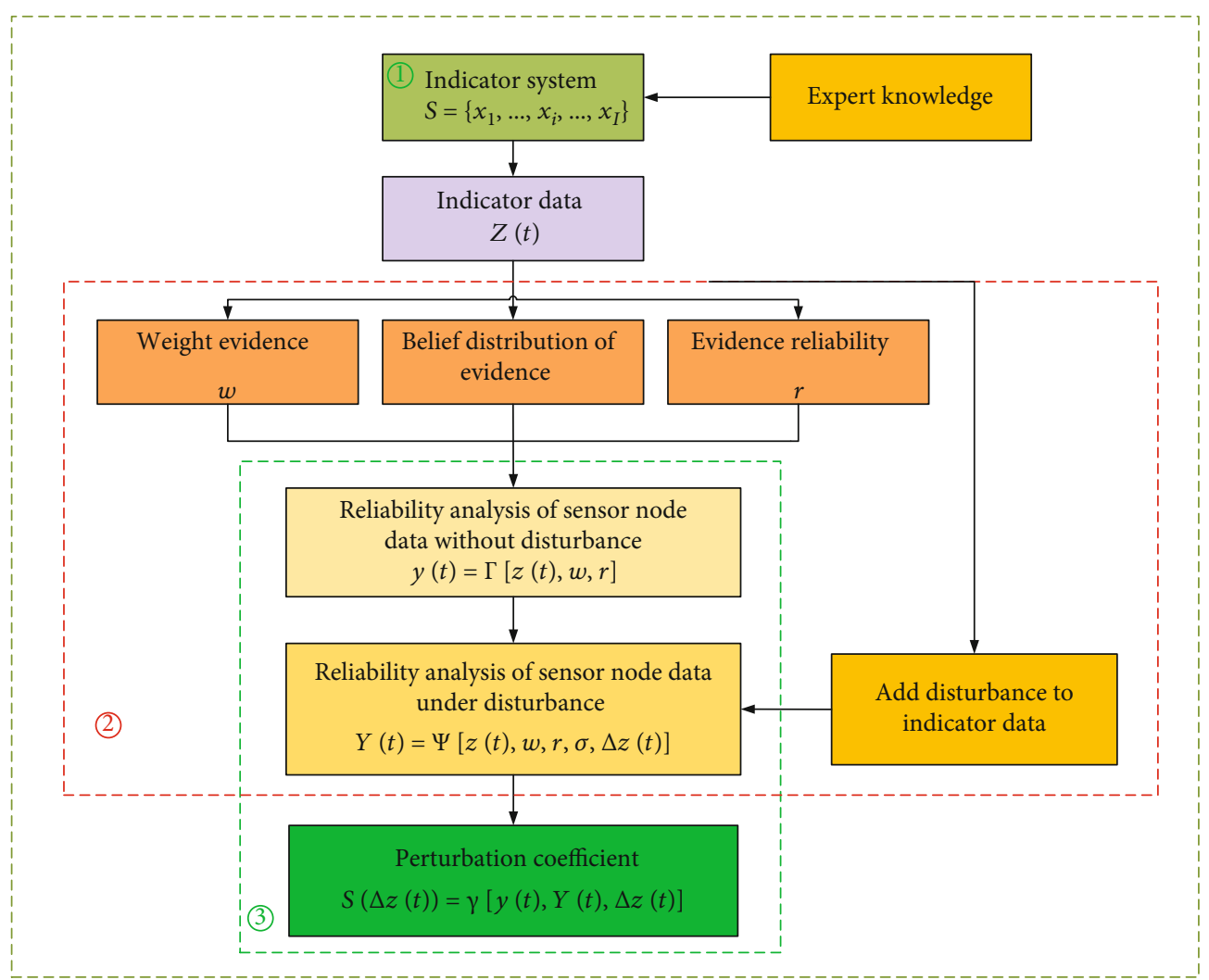

FIGURE 1: Data reliability evaluation of sensor nodes considering perturbation.

sional sensor data set about indicator $x_{i}$ collected by node $m$ in a sliding time window $[t-n+1, t]$.

$$
\xi=\sqrt{\frac{1}{\mathrm{n}-1} \sum_{k=0}^{n-1}\left[a_{i}^{m}(t-k)-\overline{A_{i}^{m}(t)}\right]^{2}}
$$

where $\xi$ is the standard deviation corresponding to data set $A_{i}^{m}(t) . \overline{A_{i}^{m}(t)}=\sum_{k=0}^{n-1} a_{i}^{m}(t-k) / n$ is the mean value of $n-D i$ mensional data in set $A_{i}^{m}(t)$.

3.1.2. Spatial Correlation. The monitoring environment is similar between different nodes in the neighborhood. The measured environmental attribute values should be similar and have the same changing trend. Therefore, in this paper, absolute error is used to reflect data proximity between different nodes. Besides, the Pearson correlation coefficient is used to reflect data trends among nodes. This is regarded as the spatial correlation of nodes.

(1) Pearson Correlation Coefficient. Pearson correlation coefficient [29] is used to express the statistical indicator of the close relationship between variables in a period. The correlation coefficient is expressed as follows:

$$
\chi_{l, m}(t)=\frac{\operatorname{cov}(l, m)}{\sqrt{\operatorname{var}(l) \operatorname{var}(m)}}
$$

where the range of correlation coefficient $\chi_{l, m}(t)$ is $[-1,1]$. 1 means that two variables are entirely linearly related. -1 means that the two variables are completely negatively correlated. The closer the data is to 0 , the weaker the correlation and 0 means that the two variables are not related.

$$
\begin{aligned}
\operatorname{var}(m) & =\frac{\sum_{k=0}^{n-1}\left[a_{i}^{m}(t-k)-\overline{A_{i}^{m}(t)}\right]^{2}}{n-1} \\
\overline{A_{i}^{m}(t)} & =\frac{\sum_{p=0}^{n-1} a_{i}^{m}(t-p)}{n}
\end{aligned}
$$

where $\operatorname{var}(m)$ represents the variance corresponding to the sensor data set $A_{i}^{m}(t) . \overline{A_{i}^{m}(t)}$ represents the mean value of the $n$-Dimensional data in the set $A_{i}^{m}(t)$.

It is known that adjacent node $l, m$ obtains $n$-Dimensi onal data sets of $A_{i}^{l}(t)$ and $A_{i}^{m}(t)$ at time $t$, respectively. The covariance $\operatorname{cov}(l, m)$ of the data set of nodes $l, m$ is calculated as follows:

$$
\operatorname{cov}(l, m)=\frac{\sum_{k=0}^{n-1}\left[a_{i}^{l}(t-k)-\overline{A_{i}^{l}(t)}\right]\left[a_{i}^{m}(t-k)-\overline{A_{i}^{m}(t)}\right]}{n-1}
$$


(2) Absolute Error. Absolute error is used to calculate the proximity of node data to other nodes in the neighborhood. The calculation formula is

$$
\begin{aligned}
\Phi_{m}(t) & =\left|\overline{A_{i}^{m}(t)}-\overline{A_{i}(t)}\right| \\
\overline{A_{i}(t)} & =\frac{\sum_{q=1}^{Q} \overline{A_{i}^{q}(t)}}{Q-1}(q \neq m)
\end{aligned}
$$

where $\Phi_{m}(t)$ represents the absolute error between node $m$ and other nodes in a certain time window $[t-n+1, t]$. $\overline{A_{i}(t)}$ represents the average value of indicator $x_{i}$ collected by all nodes in the neighborhood, except node $m$. There are $Q$ nodes in this neighborhood.

3.2. Evaluation Indicator Weight and Reliability Calculation. Before using ER rule to fuse indicator data, we need to get the weight of evidence and reliability parameters. In this paper, the coefficient of variation method and the distance-based method is used to determine indicator weight and data reliability [30]:

3.2.1. Indicator Weight. The indicator weight represents the relative importance of the indicator information in the reliability evaluation of wireless sensor networks. The coefficient of variation method gives the indicator weight by judging the relative change range of the indicator data. Let the weight of the indicator be $w_{1}, w_{2}, w_{3}$. The indicator weight is

$$
w_{i}=\frac{v_{z_{i}}}{\sum_{i=1}^{n} v_{z_{i}}},(n=3)
$$

Among them, $v_{z_{i}}=s_{z_{i}} / \overline{z_{i}}, s_{z_{i}}$ is the sample mean square error of index $x_{i}$ in time $H, s_{z_{i}}=\sqrt{1 /(H-1) \sum_{h=1}^{H}\left(z_{i}(h)-\overline{z_{i}}\right)^{2}} \cdot z_{i}(h)$ represents the data of the indicator $x_{i}$ at time $h . \overline{z_{i}}$ is the sample mean value. $\overline{z_{i}}=1 / H \sum_{h=1}^{H} z_{i}(h) . v_{z_{i}}$ is the change range of the indicator. In WSN, due to the characteristics of its own stability, the larger the change range of the indicator, the greater the ability of the indicator to reflect abnormal data, and the greater the weight, which meets the actual requirements.

3.2.2. Indicator Reliability. Indicator reliability is used to represent the ability of indicator data to describe the real characteristics of wireless sensor networks, which is an objective attribute. It can be expressed by the average distance of indicator data. The reliability of the indicator is assumed to be $r_{1}$, $r_{2}$ and $r_{3}$, respectively. From a distance-based method, the following results are obtained:

$$
\begin{aligned}
& d_{i, h}=\left|z_{i}(h)-\overline{z_{i}}\right| \\
& \overline{D_{i, h}}=\frac{1}{H} \sum_{h=1}^{H} d_{i, h}=\frac{1}{H} \sum_{h=1}^{H}\left|z_{i}(h)-\overline{z_{i}}\right|
\end{aligned}
$$

where $z_{i}(h)$ represents the data of the index $x_{i}$ at time $h . \overline{z_{i}}$ is the average value of all index data in time $H . d_{i, h}$ is the dis- tance between $z_{i}(h)$ and $\overline{z_{i}} \cdot \overline{D_{i, h}}$ is the average distance of all test data. The indicator reliability is:

$$
r_{i}=\frac{\overline{D_{i, h}}}{\max d_{i, h}}
$$

In the above formula, $d_{i, h}$ is used to represent the volatility of the test data. Based on the analysis of the wireless sensor network's stability characteristics, the greater the fluctuation, the lower the reliability, and the smaller the $r_{i}$.

3.3. Indicator Data Standardization. Standardization of indicator data is the basis for evaluation issues. When the data is unified, the synthesis of indicators is meaningful. According to the analysis of historical engineering test and the understanding of WSN working mechanism, the indicator reference level and reference value are determined by experts. Then, according to the reference level and reference value, we use the rule-based information transformation method [23] to transform the indicator data into belief distribution. The belief degree $p_{i, j}$ of the input value can be calculated as follows:

$$
\left\{\begin{array}{l}
p_{i, j}=\frac{h_{i, j+1}-z_{i, j}}{h_{i, j+1}-h_{i, j}}, h_{i, j} \leq z_{i, j} \leq h_{i, j+1}, j=1, \cdots, J-1 \\
p_{i, j+1}=1-p_{i, j}, h_{i, j} \leq z_{i, j} \leq h_{i, j+1}, j=1, \cdots, J-1 \\
p_{i, k}=0, k=1, \cdots, J ; k \neq j, j+1
\end{array}\right.
$$

where $h_{i, j}(i=1,2, \cdots, I ; j=1,2, \cdots, J)$ is the reference value of indicator $x_{i} . J$ represents the number of reference values, and $h_{i, j+1} \geq h_{i, j} . z_{i, j}$ represents the input data of indicator $x_{i}$.

3.4. Reliability Evaluation Method of Sensor Node Data Based on Evidential Reasoning Rules in a Disturbed Environment

3.4.1. Basic Structure of Data Reliability Analysis for Wireless Sensor Nodes considering Disturbance. Based on standardizing indicator data and calculating its weight and reliability, ER rule is used to fuse indicator data and parameters. It is used to calculate the data reliability of sensor nodes at a specific time. According to the calculation formula of ER rule, the implementation process of ER rule is analyzed in detail. Suppose that a node collects $\mathrm{T}$ pieces of data, and each piece of information has $I$ indicators. The input data is $z_{i}(i=1$, $\cdots, I)$, which is represented as evidence $e_{i}(i=1, \cdots, I)$. The frame of discernment is composed of $N$ evaluation level $H_{n}$ $(n=1, \cdots, N)$, namely $\Theta=\left\{H_{1}, \cdots, H_{N}\right\}$. After data standardization, the evidence can be expressed as the following form of belief distribution:

$$
e_{i}=\left\{\left(H_{n}, p_{n, i}\right), n=1, \cdots, N ;\left(\Theta, p_{\Theta, i}\right)\right\}
$$

where $p_{n, i}$ is the belief degree of the evaluation scheme evaluated as evaluation level $H_{n}$ under evidence $e_{i}$. $p_{\Theta, i}$ represents the belief degree of the ith indicator relative to the discernment framework $\Theta$, namely global ignorance, and satisfies 0 
$\leq p_{n, i} \leq 1, \sum_{n=1}^{N} p_{n, i} \leq 1$. The weight of evidence is $w_{i}(i=1$, $\cdots, I)$, which satisfies $0 \leq w_{i} \leq 1$ after normalization. The reliability of the evidence is $r_{i}(i=1, \cdots, I)$, and it satisfies $0 \leq r_{i}$ $\leq 1$. Then its mixed weighted belief distribution form is:

$$
m_{i}=\left\{\left(H_{n}, \tilde{m}_{n, i}\right), \forall \theta \subseteq \Theta ;\left(p(\Theta), \tilde{m}_{p(\Theta), i}\right)\right\}
$$

where $P(\Theta)$ is a power set. $\tilde{m}_{n, i}$ represents the mixed probability quality of the ith indicator under level $H_{n}$, and satisfies:

$$
\tilde{m}_{n, i}= \begin{cases}0, & H_{n}=\varnothing \\ c_{r w, i} m_{n, i}, & H_{n} \subseteq \Theta, H_{n} \neq \varnothing \\ c_{r w, i}\left(1-r_{i}\right), & H_{n}=P(\Theta)\end{cases}
$$

where $\varnothing$ is an empty set. $c_{r w, i}=1 /\left(1+w_{i}-r_{i}\right)$ is the normalization coefficient. $m_{n, i}$ is the basic probability quality of the ith indicator under level $H_{n}$, and $m_{n, i}=w_{i} p_{n, i}$.

Each indicator is used to describe some aspect of the physical environment. The data of each indicator of a node can be obtained by Equation (15) and its belief distribution can be expressed by Equation (16). The final reliability evaluation result is obtained by fusing the indicator information through ER rules. The combined belief $p_{\theta, e(I)}$ of $\theta$ is determined by the following formula for $I$ piece of evidence:

$$
\begin{aligned}
& \widehat{m}_{n, e(k)}=\left[\left(1-r_{k}\right) m_{n, e(k-1)}+m_{p(\Theta), e(k-1)} m_{n, k}\right] \\
& +\sum_{A \cap B=\theta} m_{A, e(k-1)} m_{B, k} \\
& \widehat{m}_{p(\Theta), e(k)}=\left(1-r_{k}\right) m_{p(\Theta), e(k-1)} \\
& m_{\theta, e(k)}=\left\{\begin{array}{l}
0, H_{n}=\varnothing \\
\frac{\widehat{m}_{n, e(k)}}{\sum_{D \subseteq \Theta} \widehat{m}_{D, e(k)}+\widehat{m}_{P(\Theta), e(k)}}, H_{n} \neq \varnothing
\end{array}\right. \\
& p_{\theta, e(k)}=\left\{\begin{array}{l}
0, H_{n}=\varnothing \\
\frac{\widehat{m}_{n, e(k)}}{\sum_{D \subseteq \Theta} \widehat{m}_{D, e(k)}}
\end{array}, H_{n} \subseteq \Theta, H_{n} \neq \varnothing\right.
\end{aligned}
$$

Among them, $k=1,2, \cdots, I, \quad p_{\theta, e(k)}$ represents the belief degree of the first $k$ indicators to the evaluation level $H_{n}$ after fusion and meets the requirements of $m_{n, e(1)}=m_{n, 1}$ and $m_{p(\theta), e(1)}=m_{p(\theta), 1}$. Through the above iterative algorithm, the comprehensive evaluation results are obtained:

$$
e(I)=\left\{\left(H_{n}, p_{n, e(I)}\right), n=1, \cdots, N,\left(\Theta, p_{\Theta, e(I)}\right)\right\}
$$

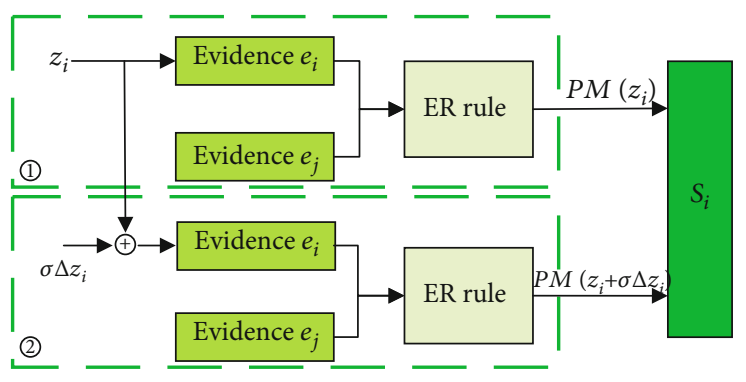

FIgURE 2: WSN node data reliability model considering perturbation.

Let the utility of evaluation level $H_{n}$ be $u\left(H_{n}\right)$. The expected utility of the evaluation scheme is calculated by the utility-based method [23].

$$
u=\sum_{n=1}^{N} u\left(H_{n}\right) p_{n, e(I)}+u(\Theta) p_{\Theta, e(I)}
$$

where $u$ is the expected utility of the evaluation, which can be used to describe the data reliability of wireless sensor networks.

However, some factors will affect the reliability of data collection and analysis, and reduce the real scene's application quality. Such as network fluctuations, environmental confrontation, and other factors. Therefore, we consider introducing disturbance effects in the data. As shown in the figure below:

In Figure 2, the first part is the original sample of data reliability evaluation, with two pieces of evidence $e_{i}$ (input data is $z_{i}$ ) and $e_{j}$ as examples. The ER rule is used to fuse the two parts of evidence. The data reliability evaluation result of the node can be obtained, which is recorded as $\operatorname{PM}\left(z_{i}\right)$. Where $\operatorname{PM}\left(z_{i}\right)$ means the utility of data $z_{i}$, which is essentially the same as expected utility $u$. In the second part, the disturbance samples are considered. Based on the original sample, the input data of evidence $e_{i}$ becomes evidence $\left(z_{i}+\sigma \Delta z_{i}\right)$. Where $\sigma$ is the disturbance intensity. $\Delta z_{i}$ is the disturbance variable. The reliability evaluation result of node data is obtained under the condition of disturbance, which is recorded as $\operatorname{PM}\left(z_{i}+\sigma \Delta z_{i}\right)$. The belief distribution of evidence $e_{i}$ is obtained under disturbance situation by Equation (15).

$e_{i}=\left\{\left(H_{l}, \frac{z_{i}+\sigma_{i} \Delta z_{i}-h_{l+1}}{h_{l}-h_{l+1}}\right),\left(H_{l+1}, \frac{h_{l}-z_{i}-\sigma_{i} \Delta z_{i}}{h_{l}-h_{l+1}}\right),\left(H_{k}, 0\right)\right\}$

Among them, $k \in[1, N]$ and $k \neq l, l+1$.

The other indicators are known. And the rule-based method is used to transform it into the form of belief distribution. According to ER rule, sensor nodes' data reliability considering disturbance can be obtained by fusing the indicator information. To further analyze the adjustment ability of nodes to different disturbance signals and measure the 


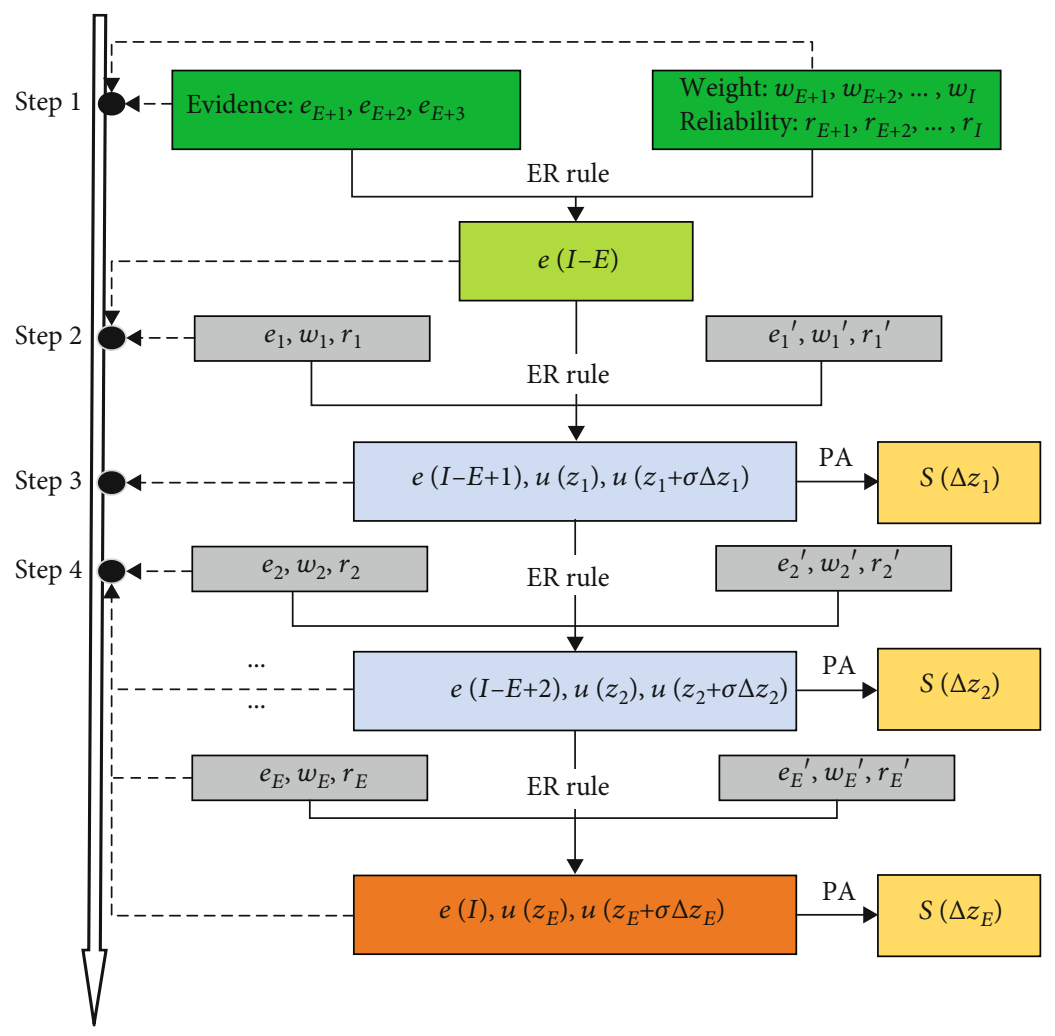

FIGURE 3: Reasoning process of ER rule-PA.

adaptability of nodes to disturbance environment, disturbance factor $S_{i}$ is proposed:

$$
S_{i}=\frac{P M\left(z_{i}+\sigma \Delta z_{i}\right)-P M\left(z_{i}\right)}{\Delta z_{i}}
$$

If $S_{i}<|\varepsilon|$, it means that the impact on the node is acceptable in this environment. The node can complete the job successfully. $\varepsilon$ is the maximum error of the disturbance factor.

3.4.2. Inference Process of Data Reliability Analysis of Wireless Sensor Nodes considering Disturbance. In the last part, the mathematical model has been established for the implementation of the evidence reasoning rule considering disturbance. In this section, the general method of the model is analyzed in detail. The original data is added disturbance. According to Equation (26), if $\sigma=0$ or $\Delta z_{i}=0$, then $u\left(z_{i}+\right.$ $\left.\sigma \Delta z_{i}\right)=u\left(z_{i}\right)$, we get $S\left(\Delta z_{i}\right)=0$, which means the disturbance is invalid. The implementation of ER rule follows exchangeability, that is, the combined order of evidence will not affect the final result. Based on this feature, the implementation of ER rule-PA is divided into four steps, as shown in Figure 3. The following is the reasoning process of ER rule-PA.

Step 1: By fusing $(I-E)$ piece of evidence with invalid disturbance, we get the result $e(I-E)$ as shown in Equation (23).

Step 2: The first disturbance evidence $e_{1}{ }^{\prime}$ is fused with the result of the first step to get $e(I-E+1)$. And the expected utility $u\left(z_{1}+\sigma \Delta z_{1}\right)$ under disturbance and the expected utility $u\left(z_{1}\right)$ without disturbance are calculated, respectively.
Step 3: The disturbance factor $S\left(\Delta z_{1}\right)$ is calculated according to Equation (26).

Step 4: The second and third steps are repeated to fuse the residual disturbance evidence with $e(I-E+1)$. And the disturbance factors are calculated in turn.

\section{Case Analysis}

The validity of the above model is verified in this chapter. By analyzing the indicator of sensor node data, ER rules are used to evaluate sensor node data reliability. Moreover, sensor nodes' network stability and environment antagonism are introduced as the main disturbance factors. The influence on sensor nodes is considered in a disturbed environment.

4.1. Background of the Experiment. The experimental data of this paper comes from the wireless sensor experiment of the Intel Berkeley research laboratory. In this experimental scenario, some modules are installed in sensor nodes, such as temperature sensing, humidity sensing, light sensing, etc. The experimental environment can be real-time monitored to obtain the temperature, humidity, light intensity, and other environmental data of the target environment. As shown in Figure 4. In this paper, through the measured data collected by the sensor, 169239 pieces of data are sampled as experimental data from four adjacent nodes in WSN within 30 days. However, due to the different time interval of data collection, and useless data, the experimental data is processed. After processing, each node has 3030 pieces of data, a total of 12120 pieces of data, in which each environmental 


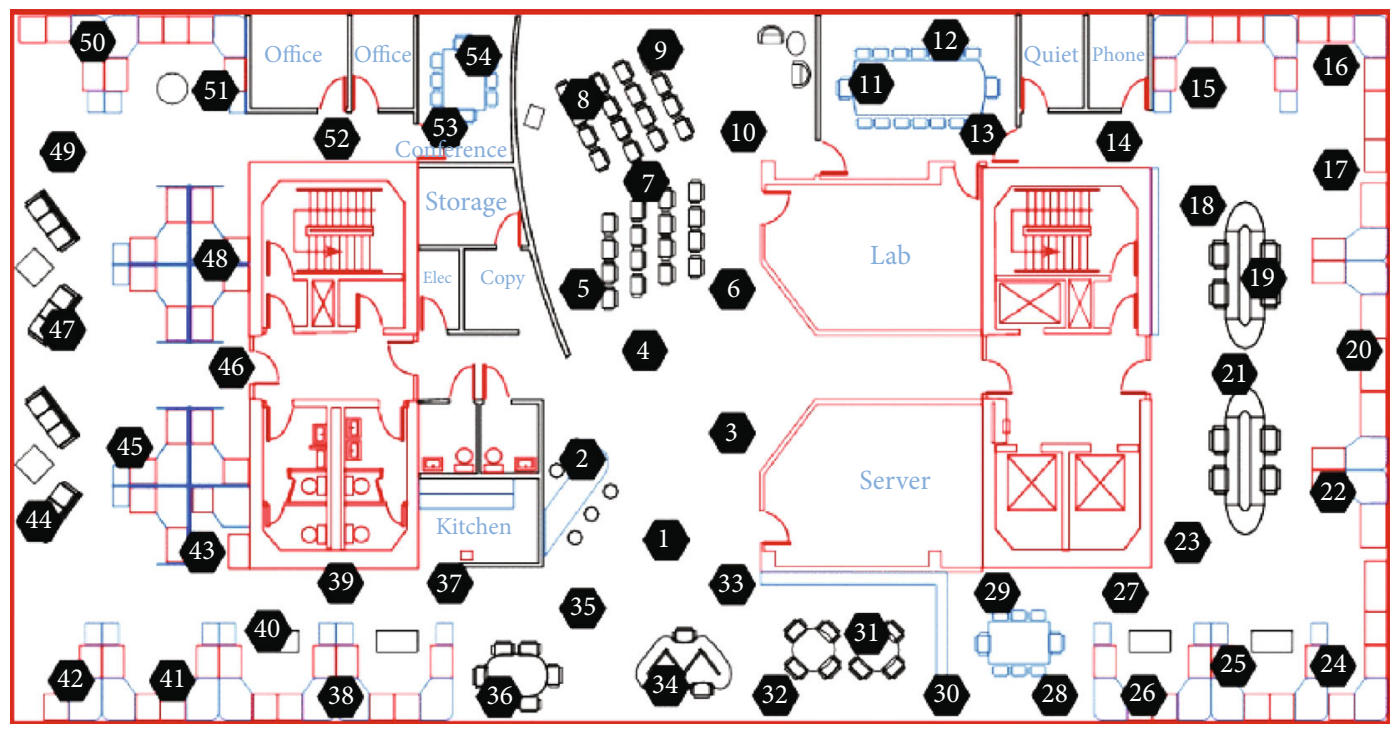

FIGURE 4: Distribution map of wireless sensors at Intel Berkeley Research Lab.

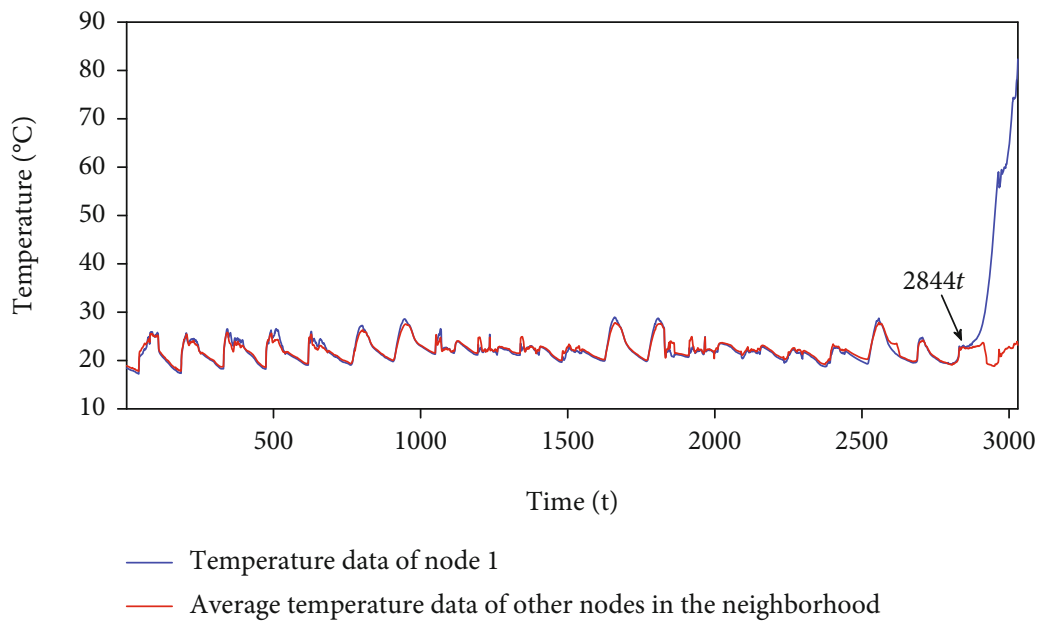

Figure 5: Temperature comparison between node 1 and other nodes.

data is the average value of sampling within 10 minutes. Considering the space, temperature data are used as the main experimental data for evaluation in this paper.

4.2. Indicator Data. Node 1 is used as an example in this paper. In Figure 5, the temperature data of node 1 and the average temperature data of other nodes in the neighborhood are shown without disturbance. Considering the actual working state of each node in the sensor, the temperature threshold is set according to the actual temperature. Once the temperature data of the node exceeds the threshold at a particular time point, and the threshold cannot be corrected. Then it can be judged that the node is faulty, and the node is not considered. From the analysis of Figure 1, it can be concluded that the temperature data of node 1 has a high degree of coincidence with the data of neighboring nodes. And there are obvious differences in a few time points. Moreover, the temperature of node 1 increased sharply after $2844 \mathrm{t}$.
TABLE 1: Indicator evaluation level and reference value.

\begin{tabular}{lccc}
\hline & $H_{1}$ & $H_{2}$ & $H_{3}$ \\
\hline Standard deviation & 0 & 0.5 & 1.5 \\
Absolute error & 0 & 0.5 & 2 \\
Correlation coefficient & 1 & 0.7 & 0 \\
\hline
\end{tabular}

4.3. Data Reliability Evaluation of Wireless Sensor Networks Based on ER Rule under Disturbed Environment

4.3.1. Data Reliability Analysis of Wireless Sensor Networks without Disturbance. Based on the model proposed in Section 3.4, the data reliability of sensor nodes is evaluated. According to the expert experience and the sensor's working mechanism, the evaluation level is set as "high", "medium", and "low". Therefore, the discernment frame is $\Theta=\left\{\left(\mathrm{H}_{1}, h\right.\right.$ igh $),\left(\mathrm{H}_{2}\right.$, medium $),\left(\mathrm{H}_{3}\right.$, low $\left.)\right\}$. And the reference values 

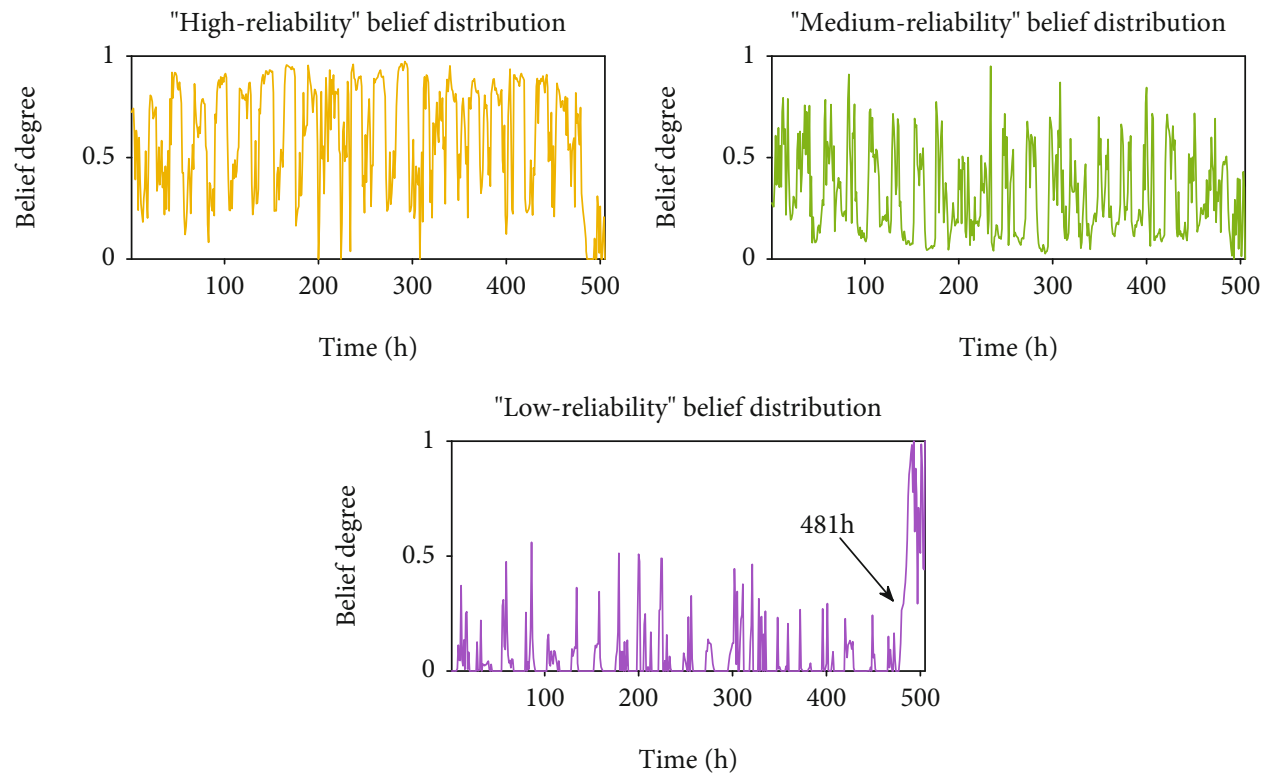

FIGURE 6: Reliability evaluation results of No. 1 node in WSN.

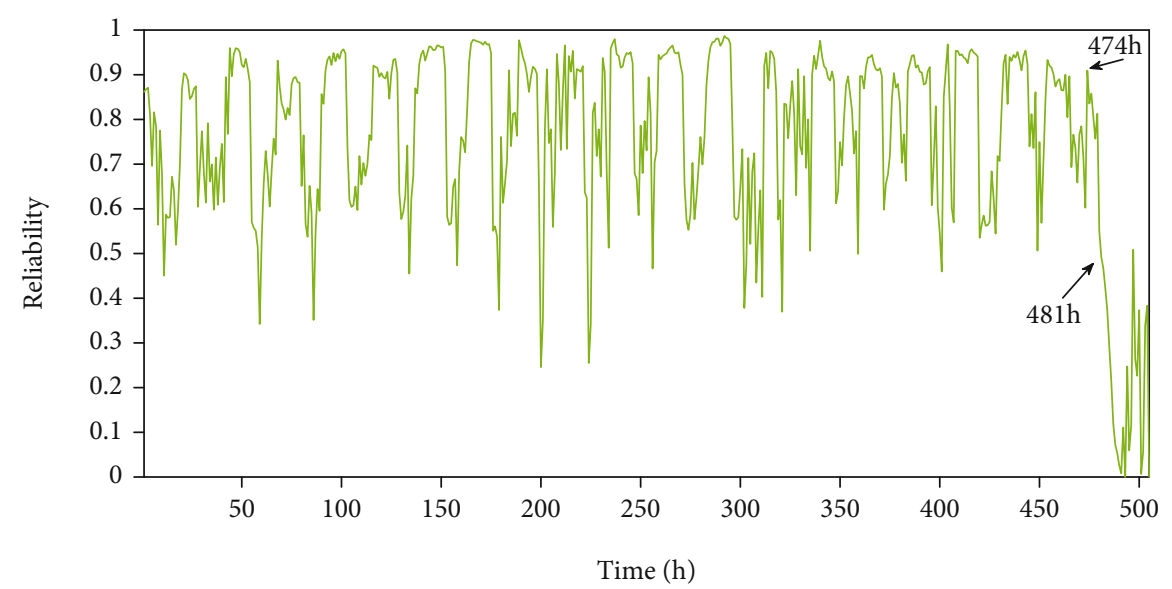

FIGURE 7: Reliability evaluation result of single node in WSN.

are set as shown in Table 1. The "medium" reference value of standard deviation and the absolute error is the mode of the eigenvalue. And the "low" reference value is set to the maximum number. The reference value of correlation coefficient is divided by reasonable partition, according to the correlation level and expert knowledge.

Disturbance analysis is an experimental method by adding a disturbance sample trajectory on the basis of the original data. It is used to analyze the sensitivity of the system performance indicator to a critical parameter. In this paper, the undisturbed situation of indicator data is analyzed as follows. Suppose that the time sliding window is 1 hour. And the unit is h. Therefore, 3030 sets of data are set to 5056 dimensional vectors. The time dependence of temperature is obtained by Equation (5) and (6). And the space dependence of temperature is obtained by Equation (7)-(10). The data is transformed by a rule-based method as shown in Equation (15). Then the data is transformed into the form of belief distribution as shown in Equation (16). The following is an example of belief transformation: Suppose that the correlation coefficient of indicator temperature is 0.9 at time $t$, $p_{1,1}(t)=(0.9-0.7) /(1-0.7)=0.67 p_{1,2}(t)=1-(0.9-0.7) /$ $(1-0.7)=0.33, p_{1,3}(t)=0$. Therefore, the belief distribution of Equation (16) can be expressed as:

$$
e_{i}(t)=\left\{\left(H_{1}, 0.67\right),\left(H_{2}, 0.33\right),\left(H_{3}, 0\right)\right\}
$$

The weights of standard deviation, absolute error and correlation coefficient are $w_{1}, w_{2}$ and $w_{3}$, respectively. And the reliability is $r_{1}, r_{2}$ and $r_{3}$, respectively. In the following, we use the coefficient of variation method as shown in Equation (11) to obtain the weights of the indicators, which are $w_{1}=0.38, w_{2}=0.41$ and $w_{3}=0.21$, respectively. According to the Equation (12)-(14), the indicator reliability is $r_{1}=0.7$ , $r_{2}=0.67, r_{3}=0.71$. The reliability of node 1 is evaluated 

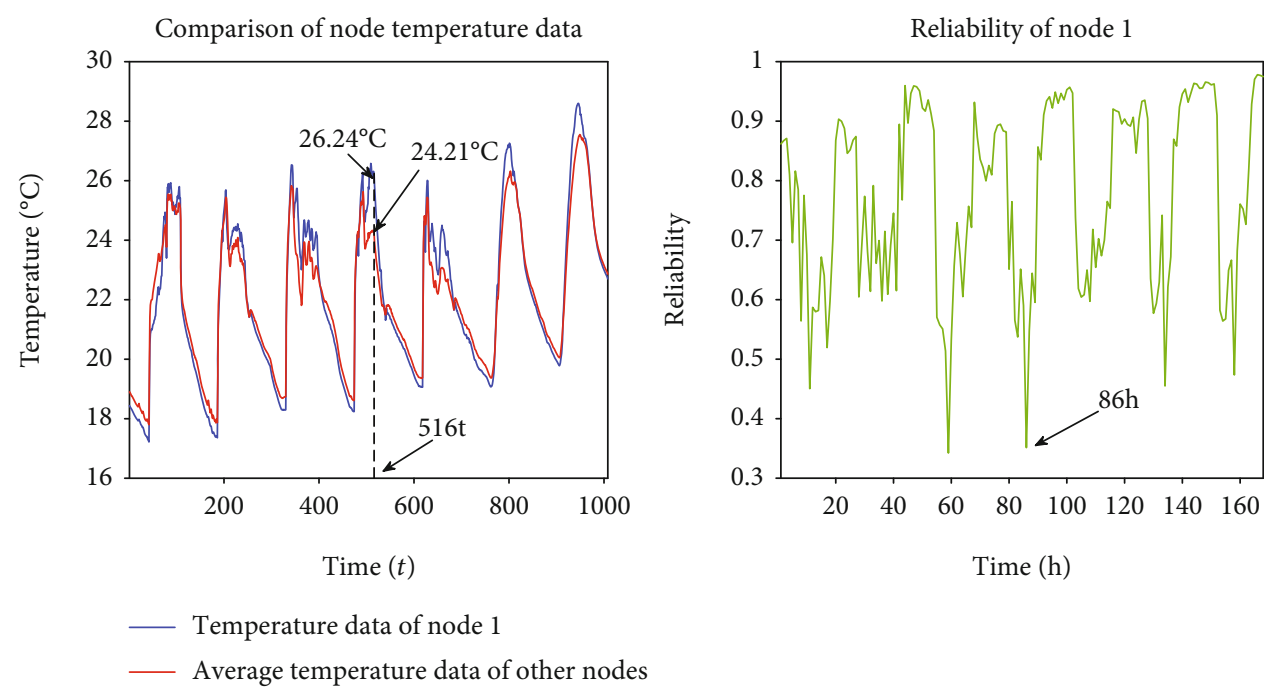

FIgURE 8: Partial temperature comparison and corresponding time reliability.
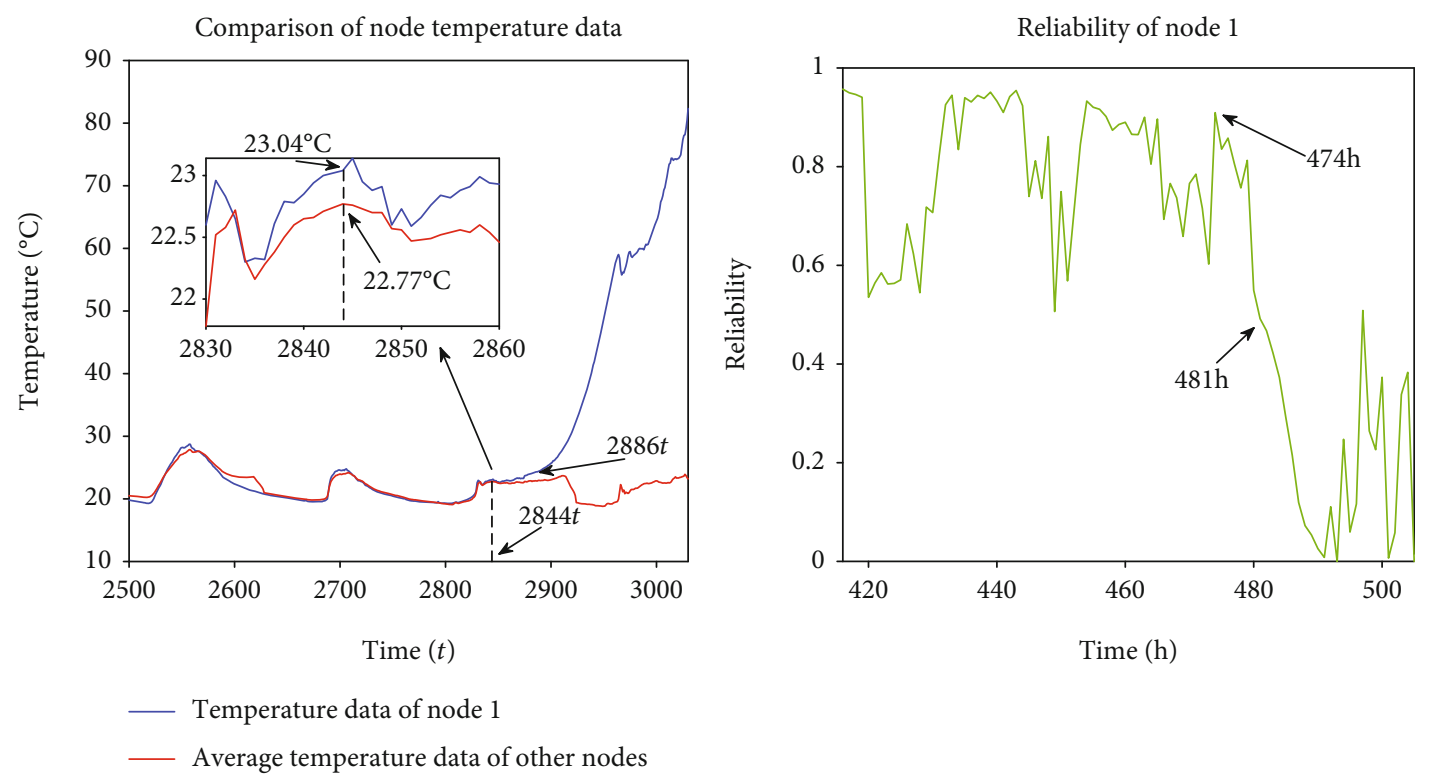

FIgURE 9: Partial temperature comparison and corresponding time reliability.

by using ER rules as Equation (19)-(23). And the evaluation results are shown in Figure 6:

It can be seen from the above figure that the data reliability of node 1 is mostly concentrated in "high" reliability in the process of environment detection. The second is "medium" reliability. And "low" reliability is less, mainly distributed after $481 \mathrm{H}$. The above analysis is consistent with the real situation of the sensor. The overall situation remained in the good state.

Among them, $u\left(H_{1}\right)=1, u\left(H_{2}\right)=0.5$ and $u\left(H_{3}\right)=0$ are set. As shown in Figure 7, the expected utility of reliability is calculated by using the utility-based method as shown in Equation (24).

In Figure 7, the expected utility of the reliability evaluation results of node 1 ranges from 0.5 to 1 . And this belongs to the middle and high levels. Among them, the reliability of a few instantaneous time points is less than 0.5 . It can be seen from Figure 7 that the reliability decreases after $474 \mathrm{~h}$. After $481 \mathrm{~h}$, the node reliability decreases and remains below 0.5 . This indicates that the node has failed.

To verify the effectiveness of the method, the points with partial reliability lower than 0.5 will be selected and compared with Figure 5. The time points of abnormal fluctuations in the node temperature data are accurately identified by using this method. As shown in Figures 8 and 9 .

In Figure 8, abnormal data and reliability of nodes are displayed in normal working time. For example, the temperature difference and the temperature change trend of the nodes in the neighborhood are quite different at $516 \mathrm{t}$. And the corresponding node reliability is lower than 0.5 at $86 \mathrm{~h}$. 

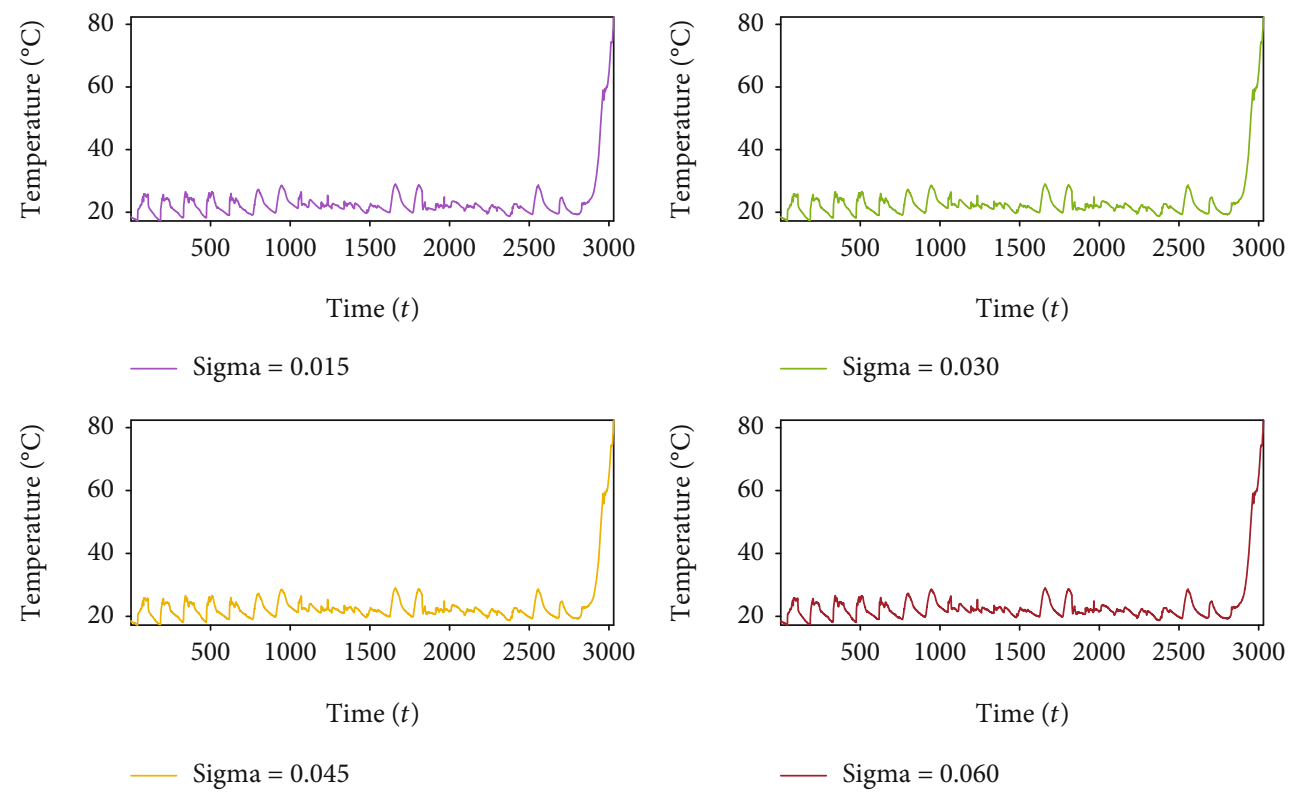

Figure 10: Temperature data under different perturbation intensities.

In Figure 9, temperature data and reliability are displayed at the time of node failure. After $2844 \mathrm{t}$, the temperature data of node 1 is very different from the average temperature data of other nodes. Corresponding, the reliability began to decrease after $474 \mathrm{~h}$. After $481 \mathrm{~h}$, the reliability is less than 0.5 .

After $2844 \mathrm{t}$, the difference of temperature data gradually decreased and then increased over a period of time in Figure 9. This is because the evaluation result of $474 \mathrm{~h}$ is the average evaluation result of data change in the period of $2838 \mathrm{t}$ to $2844 \mathrm{t}$. Therefore, compared with other time in the critical interval, the evaluation result here is better. It indicates that the node is invalid. The evaluation results are consistent with the actual situation. The validity of reliability evaluation results is proved.

4.3.2. Reliability Analysis of Sensor Node Data under Disturbance. After analyzing the data reliability of sensor nodes by ER rules, the real-time state of sensor node reliability can be observed. But the disturbance of external factors to sensor nodes is not considered. In addition, through the analysis of the sensor's working model, it is found that it will inevitably be affected by various factors. In this section, we evaluate the reliability of sensor nodes based on the abovementioned data reliability evaluation model considering disturbance. The disturbance variable is added to the nominal trajectory to simulate the node's working state affected by different factors. And the disturbance of different intensity is set. The disturbance variable is the sensor node's actual data relative to the perception information in an undisturbed environment. It has the following characteristics:

(1) The generation of disturbance is random and irregular

(2) The generation of disturbance variables accords with the characteristics of normal distribution
TABLE 2: Indicator weights under different perturbation intensities.

\begin{tabular}{lcccc}
\hline & $\sigma=0.015$ & $\sigma=0.030$ & $\sigma=0.045$ & $\sigma=0.060$ \\
\hline $\begin{array}{l}\text { Standard deviation } \\
\text { Absolute error }\end{array}$ & 0.385 & 0.385 & 0.386 & 0.388 \\
$\begin{array}{l}\text { Correlation } \\
\text { coefficient }\end{array}$ & 0.408 & 0.407 & 0.407 & 0.407 \\
\hline
\end{tabular}

TABLE 3: Indicator reliability under different perturbation intensities.

\begin{tabular}{lcccc}
\hline & $\sigma=0.015$ & $\sigma=0.030$ & $\sigma=0.045$ & $\sigma=0.060$ \\
\hline $\begin{array}{l}\text { Standard deviation } \\
\text { Absolute error }\end{array}$ & 0.699 & 0.699 & 0.698 & 0.697 \\
$\begin{array}{l}\text { Correlation } \\
\text { coefficient }\end{array}$ & 0.668 & 0.667 & 0.665 & 0.665 \\
\hline
\end{tabular}

By analyzing the disturbance factors that affect the indicator data, the accuracy of temperature data is easily affected by network fluctuations and environmental confrontation. In this paper, four types of disturbance environment are simulated, namely weak network fluctuation and weak environment countermeasure, weak network fluctuation and strong environment countermeasure, strong network fluctuation and weak environment countermeasure, strong network fluctuation and strong environment countermeasure. The corresponding disturbance intensities are $0.015,0.030,0.045$ and 0.060 , respectively. After adding disturbance, the indicator data changed. The temperature data of node 1 are listed under different disturbance intensities in Figure 10.

Nowadays, the disturbance intensity is given mainly by the subjective setting of expert knowledge. In this paper, four different values are used to characterize four different 

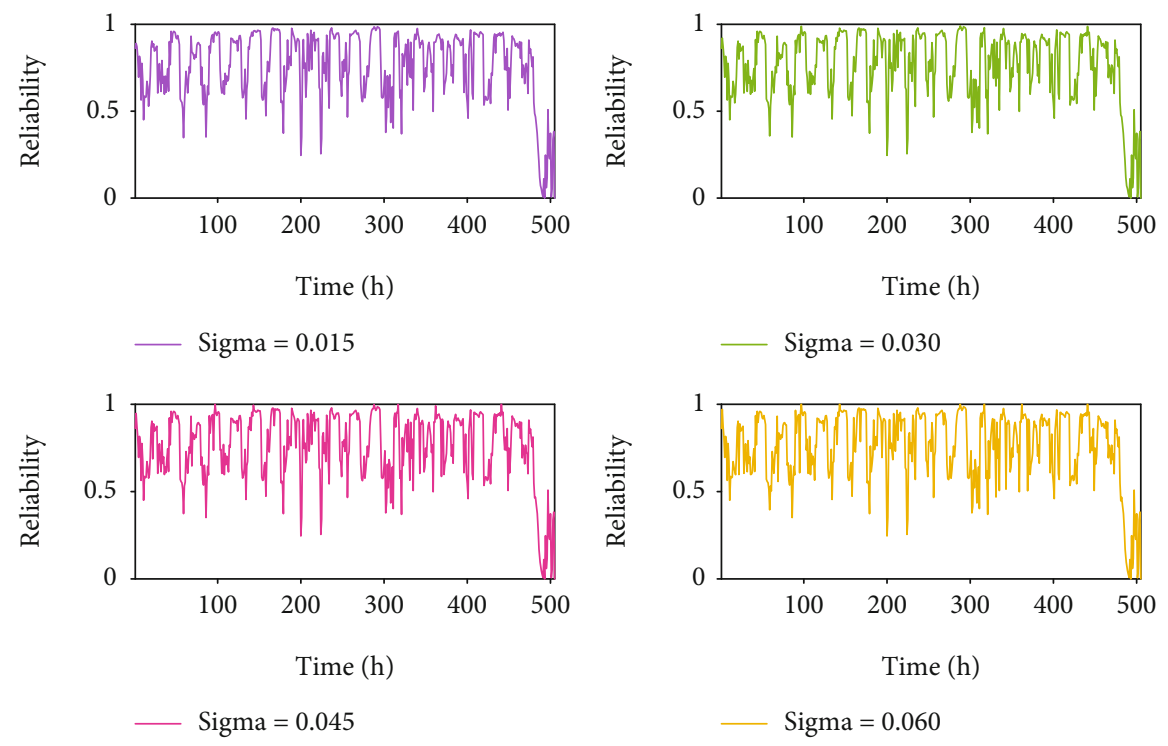

FIgURE 11: WSN node data reliability under different perturbation intensities.

disturbance environments. In the future, it is hoped that scientific calculation methods can be used to classify and accurately assign values to different disturbance environments, so as to improve the scientific of disturbance intensity. It is necessary to recalculate the indicator data of nodes with affected sensor nodes by disturbance. Moreover, due to each indicator's different stability in the disturbance environment, the weight and reliability of each indicator also change. According to Equations (11)-(14), the weight and reliability of each indicator under different disturbance intensity are calculated, as shown in Tables 2 and 3:

According to the evidential reasoning rules and utilitybased calculation method, sensor nodes' data reliability is calculated under different disturbance intensities. The expected utility of the output of evidential reasoning rules is calculated by the utility-based calculation method. This belongs to the application of decision theory, which is used to represent the comprehensive level of sensor node data reliability. As shown in Figure 11, the data reliability of nodes is shown under different disturbance intensities.

As shown in Figure 11, there are weak differences in the data reliability evaluation results of WSN nodes under different disturbance environments. However, different disturbance intensities can lead to different credibility of evaluation results. Therefore, it is necessary to consider the adaptability of WSN nodes to different disturbance environments.

Based on Equation (26), the disturbance factors of nodes with different disturbance intensities can be obtained and taken as absolute values. As shown in Figure 12, the disturbance factor reflects the node's adaptability to different disturbance environments. The smaller the disturbance factor at a specific time, the stronger and more stable the node is against the disturbance environment. Conversely, the node is damaged and needs to be repaired or replaced.

It is easy to see from the figure above that the disturbance factor increases with the increase of disturbance intensity, consistent with the actual state. The belief distribution of low reliability of WSN node data is compared under different disturbance intensity. As shown in Figure 13, when the node reliability is low, it is easier to cause the disturbance factor change; when it changes from other states to low reliability. The actual phenomenon reflected is the abnormal fluctuation of sensor node data. In other words, when WSN nodes are unstable, they are easily affected by external disturbances. Therefore, we need to consider the authenticity and availability of the data here. Suppose the maximum disturbance error $\varepsilon=30$ is set. When the disturbance intensity does not exceed 0.030 , the disturbance factor is always less than $\varepsilon$, which indicates that the node's disturbance degree is within the acceptable range. When the disturbance intensity is more excellent than 0.045 , the disturbance factor is more significant than $\varepsilon$, which indicates that the disturbance condition has interfered with the regular operation of WSN nodes. At this time, it is necessary to adjust the node or working state.

4.4. Comparative Study. ER rules belong to the expert system in essence. The expert system's function is to combine expert knowledge with objective data effectively and finally get quantitative evaluation results. To further illustrate the effectiveness of the above methods, and given the unsupervised characteristics of the experimental data, the same qualitative and quantitative analytic hierarchy process (AHP) is used to compare with this method.

First, the discriminant matrix is established. The indicators' relative importance is assigned according to the scale of 1-9 through expert knowledge.

$$
A=\left[\begin{array}{ccc}
1 & \frac{1}{3} & 2 \\
3 & 1 & 4 \\
\frac{1}{2} & \frac{1}{4} & 1
\end{array}\right]
$$



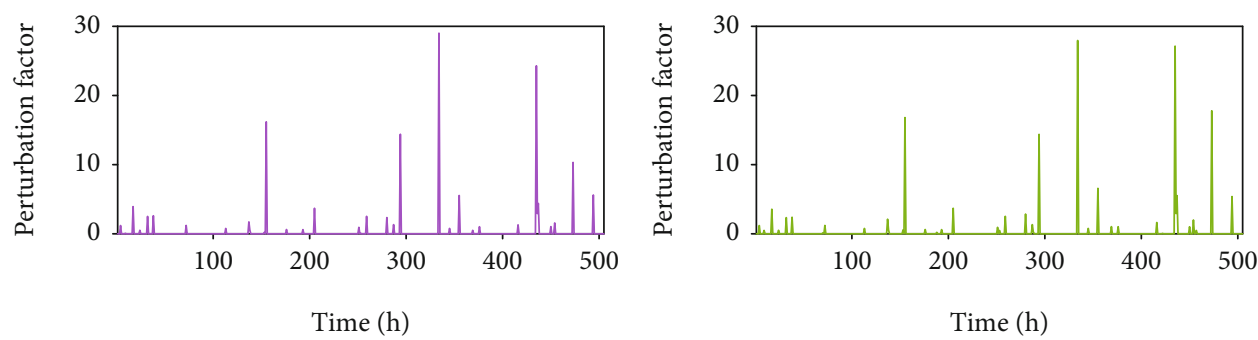

— Sigma $=0.015$

- Sigma $=0.030$
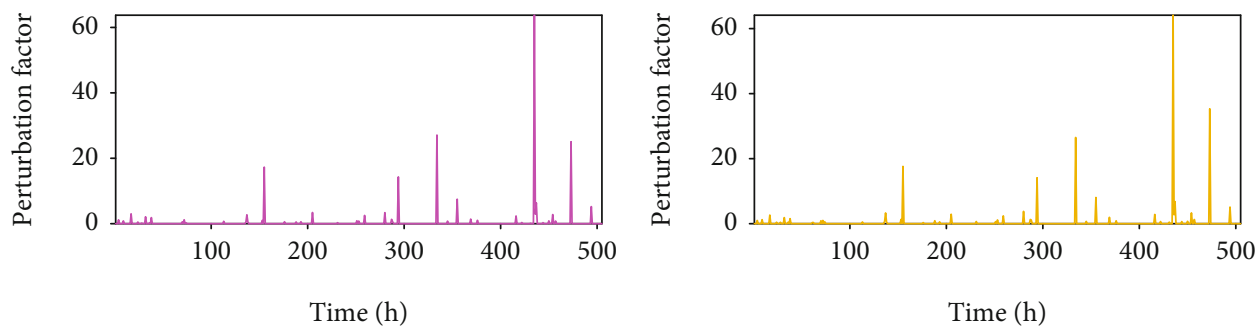

— Sigma $=0.045$

- Sigma $=0.060$

FIGURE 12: WSN node perturbation factor under different perturbation intensities.
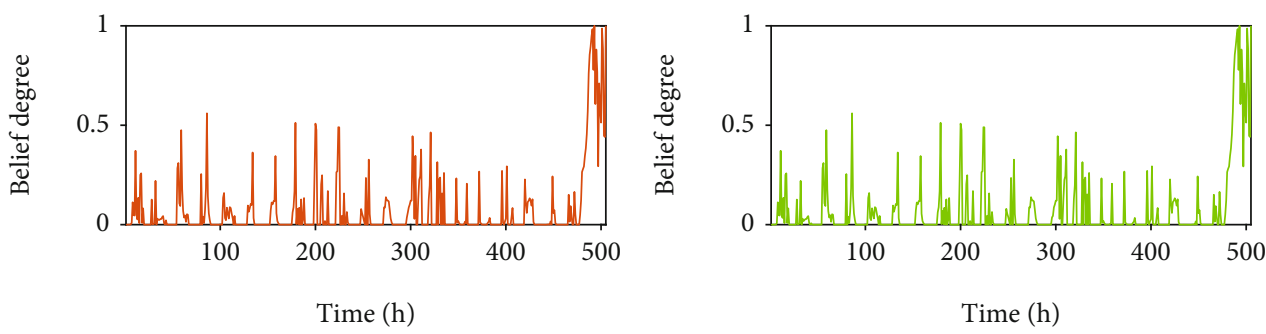

- Sigma $=0.015$

— Sigma $=0.030$
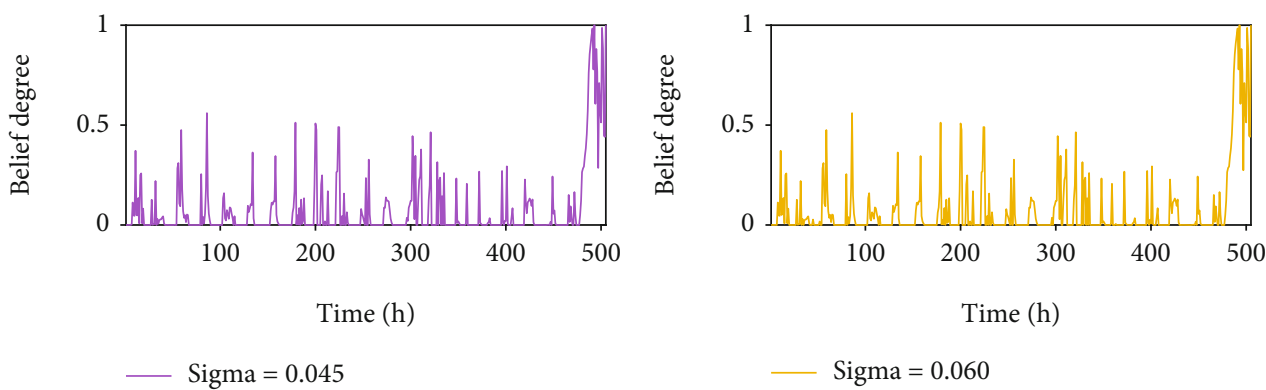

FIGURE 13: Low reliability distribution of WSN nodes under different perturbation intensities.

To verify the rationality of discriminant matrix $A$, the consistency of matrix $A$ is checked [31]. The maximum eigenvalues $\lambda_{\max }=3.0183$ and $C I=\left(\lambda_{\max }-n\right) /(n-1)=$ 0.00915 of the matrix are calculated by the square root method. $R I=0.52$ is obtained by looking up the table. The satisfactory consistency is verified as follows:

$$
C R=\frac{C I}{R I}=\frac{0.00185}{0.52}=0.0176<0.1
$$

The results show that the matrix is reasonable. The corresponding characteristic equation is obtained and normalized:

$$
W=\left[\begin{array}{lll}
0.2385 & 0.6250 & 0.1365
\end{array}\right]
$$

where $W$ is the weight vector of the evaluation indicator.

The indicator values of each time point are calculated according to the data of node and other nodes in the neighborhood. Each indicator's membership degree $x_{i}$ to the evaluation grade set $\Theta$ is transformed into a fuzzy relation matrix 
[32] as shown in Equation (31). Among them, element $b_{i, j}$ represents the membership degree of evaluation indicator $e_{i}$ relative to grade $H_{j}$. The fuzzy relation matrix can be obtained by normalizing the values, as shown in Equation (32).

$$
\begin{aligned}
& B=\left[\begin{array}{lll}
b_{11} & b_{12} & b_{13} \\
b_{21} & b_{22} & b_{23} \\
b_{31} & b_{32} & b_{33}
\end{array}\right] \\
& B=\left[\begin{array}{lll}
0.5880 & 0.2940 & 0.1180 \\
0.5560 & 0.2780 & 0.1670 \\
0.4760 & 0.3330 & 0.1900
\end{array}\right]
\end{aligned}
$$

According to Equations (30) and (32), the reliability evaluation results of sensor nodes are obtained as follows:

$$
C=W \cdot B=\left[\begin{array}{lll}
0.5527 & 0.2893 & 0.1585
\end{array}\right]
$$

From the above formula, we can get the result that the reliability of WSN data can be analyzed by the AHP method. The belief degree of "high" is 0.5527 , that of "medium" is 0.2893 , and that of "low" is 0.1585 . In the WSN data reliability distribution shown in Figure 6, "low" reliability is mainly concentrated after $481 \mathrm{~h}$. And the belief distribution of "low" and is about 0.1 . Moreover, after $505 \mathrm{~h}$, the node ultimately failed and lost the research value. The actual situation cannot be effectively described by wireless sensor reliability evaluation results based on the AHP method. And AHP method relies on expert knowledge in the process of setting parameters. Therefore, the data cannot be objectively described by the evaluation results. It can only evaluate the network as a whole. However, the model proposed in this paper combines data with expert experience. Through objective data analysis and effective use of the positive role of expert knowledge in practical engineering, it reflects the advantages of evidential reasoning rules in the fusion of multi-source information. Therefore, the proposed method is reasonable and effective in this paper.

\section{Conclusions}

In this paper, based on the analysis of the operational characteristics of WSN, taking the actual monitoring data of sensor nodes as the research object, a data reliability evaluation model of sensor nodes is proposed, which is based on ER rules. The model is used to evaluate the data reliability of WSN nodes data in no disturbance state; on this basis, the disturbance is added to ER rule to simulate the influence of different disturbance conditions on nodes in a complex working environment. Furthermore, a reliability evaluation model of sensor node data is proposed, which takes disturbance into account. In this paper, temperature time correlation and spatial correlation are used as evaluation indicators. The utility-based method is used to unify the indicator information into the form of belief distribution, which improves the expression ability of indicator information. The method of variation coefficient and distance-based method is used to obtain the weight and reliability of indicators, which overcomes the subjectivity of traditional expert weighting to a certain extent. And it improves the credibility of the consistency evaluation results. The adaptive ability of nodes to disturbance conditions is quantified by disturbance factor and maximum disturbance error. And the reliability of different disturbance environment is analyzed. The model can reasonably evaluate the data reliability of sensor nodes. And through the comparison of temperature data between nodes, the effectiveness of the model is illustrated.

\section{Data Availability}

The experimental data of this paper comes from the wireless sensor experiment of the Intel Berkeley research laboratory.

\section{Conflicts of Interest}

The authors declare that they have no conflicts of interest.

\section{Authors' Contributions}

Shukun Jin, Yawen Xie and Yanzi Gao contribute equally to this work.

\section{Acknowledgments}

This work was supported in part by the Natural Science Foundation of School of Computer Science and Information Engineering, Harbin Normal University, under Grant no. JKYKYZ202102, the Harbin Normal University pH.D. Research Start-up Gold Project under Grant XKB201905, the postgraduate practice innovation project of School of Computer Science and Information Engineering, Harbin Normal University, under Grant no. HSDSSCX2020-57, and Innovation and Entrepreneurship Project of college students in Heilongjiang Province, under Grant no. 202010231009.

\section{References}

[1] A. Boubrima, W. Bechkit, and H. Rivano, "Optimal WSN deployment models for air pollution monitoring," IEEE Transactions on Wireless Communications, vol. 16, no. 5, pp. 27232735, 2017.

[2] X. Xiao, Q. He, Z. Li, A. O. Antoce, and X. Zhang, "Improving traceability and transparency of table grapes cold chain logistics by integrating WSN and correlation analysis," Food Control, vol. 73, pp. 1556-1563, 2017.

[3] J. Q. Chen, G. Mao, C. Li, W. Liang, and D. G. Zhang, "Capacity of cooperative vehicular networks with infrastructure support: multi-user case," IEEE Transactions on Vehicular Technology, vol. 67, no. 2, pp. 1546-1560, 2018.

[4] P. Marappan and P. Rodrigues, "An energy efficient routing protocol for correlated data using CL-LEACH in WSN," Wireless Networks, vol. 22, no. 4, pp. 1415-1423, 2016.

[5] J. N. Yang, M. Ding, G. Mao, Z. Lin, D. G. Zhang, and T. H. Luan, "Optimal Base station antenna Downtilt in downlink cellular networks," IEEE Transactions on Wireless Communications, vol. 18, no. 3, pp. 1779-1791, 2019. 
[6] D. G. Zhang, J. X. Gao, X. H. Liu, T. Zhang, and D. X. Zhao, "Novel approach of distributed \& adaptive trust metrics for MANET," Wireless Networks, vol. 25, no. 6, pp. 3587-3603, 2019.

[7] W. He, G. Hu, Z. J. Zhou et al., "A new hierarchical belief-rulebased method for reliability evaluation of wireless sensor network," Microelectronics Reliability, vol. 87, pp. 33-51, 2018.

[8] P. Neng-song, Z. Wei-wei, and Z. Yu-zhao, "Anomaly detection method for wireless sensor networks based on time series data," Chinese Journal of Sensors and Actuators, vol. 31, no. 4, pp. 595-601, 2018.

[9] D. G. Zhang, G. Li, K. Zheng, X. Ming, and Z. H. Pan, “An energy-balanced routing method based on forward-aware factor for wireless sensor Networks," IEEE Transactions on Industrial Informatics, vol. 10, no. 1, pp. 766-773, 2014.

[10] H. Ge, "New multi-hop clustering algorithm for vehicular ad hoc networks," IEEE Transactions on Intelligent Transportation Systems, vol. 20, no. 4, pp. 1517-1530, 2019.

[11] T. Zhang and J. Zhang, "A kind of effective data aggregating method based on compressive sensing for wireless sensor network," EURASIP Journal on Wireless Communications and Networking, vol. 2018, no. 1, Article ID 159, p. 15, 2018.

[12] T. Zhang, D. G. Zhang, H. R. Yan, J. N. Qiu, and J. X. Gao, “A new method of data missing estimation with FNN-based tensor heterogeneous ensemble learning for internet of vehicle," Neurocomputing, vol. 420, no. 1, pp. 98-110, 2021.

[13] M. I. Alipio and N. M. C. Tiglao, "RT-CaCC: a reliable transport with cache-aware congestion control protocol in wireless sensor networks," IEEE Transactions on Wireless Communications, vol. 17, no. 7, pp. 4607-4619, 2018.

[14] D. Sharma, A. Ojha, and A. P. Bhondekar, "Heterogeneity consideration in wireless sensor networks routing algorithms: a review," The Journal of Supercomputing, vol. 75, no. 5, pp. 2341-2394, 2019.

[15] S. Liu, "Novel unequal clustering routing protocol considering energy balancing based on Network Partition \& Distance for Mobile education," Journal of Network and Computer Applications, vol. 88, no. 15, pp. 1-9, 2017.

[16] T. Zhang, T. Zhang, Y. Dong, X. H. Liu, Y. Y. Cui, and D. X. Zhao, "Novel optimized link state routing protocol based on quantum genetic strategy for Mobile learning," Journal of Network and Computer Applications, vol. 122, pp. 37-49, 2018.

[17] T. Zhang, T. Zhang, and X. Liu, "Novel Self-Adaptive Routing Service Algorithm for Application in VANET," Applied Intelligence, vol. 49, no. 5, pp. 1866-1879, 2019.

[18] S. Liu, "Novel dynamic source routing protocol (DSR) based on genetic algorithm-bacterial foraging optimization (GABFO)," International Journal of Communication Systems, vol. 31, no. 18, pp. 1-20, 2018.

[19] L. Chen and J. Zhang, "A multi-path routing protocol based on link lifetime and energy consumption prediction for mobile edge computing," IEEE Access, vol. 8, no. 1, pp. 6905869071, 2020.

[20] S. Xiang and J. Yang, "Reliability evaluation and reliabilitybased optimal design for wireless sensor networks," IEEE Systems Journal, vol. 14, no. 2, pp. 1752-1763, 2020.

[21] Y. Pan, L. Zhang, Z. W. Li, and L. Ding, "Improved fuzzy bayesian network-based risk analysis with interval-valued fuzzy sets and D-S evidence theory," IEEE Transactions on Fuzzy Systems, vol. 28, no. 99, pp. 2063-2077, 2019.
[22] L. Hao, "Evidence reasoning algorithm for multi-criteria decision-making with incomplete attribute weight information," Fire Control \& Command Control, vol. 40, no. 1, pp. 12-15, 2015.

[23] J. B. Yang and D. L. Xu, "Evidential reasoning rule for evidence combination," Artificial Intelligence, vol. 205, pp. 1-29, 2013.

[24] Y. Gong, X. Su, H. Qian, and N. Yang, "Research on fault diagnosis methods for the reactor coolant system of nuclear power plant based on D-S evidence theory," Annals of Nuclear Energy, vol. 112, pp. 395-399, 2018.

[25] Z. Fu-jun, Z. Zhi-jie, C.-h. Hu, C. Lei-Lei, and W. Li, "Online safety assessment method basedon evidential reasoning for dynamic systems," Acta Automatica Sinica, vol. 43, no. 11, pp. 1950-1961, 2017.

[26] M. Zhou, X. B. Liu, Y. W. Chen, and J. B. Yang, "Evidential reasoning rule for MADM with both weights and reliabilities in group decision making," Knowledge-Based Systems, vol. 143, pp. 142-161, 2018.

[27] Q. Liu, C. Chen, and Q. Zhang, "Perturbation analysis for total least squares problems with linear equality constraint," Applied Numerical Mathematics, vol. 161, pp. 69-81, 2021.

[28] F. Xiu-wen, Y.-s. Yang, and Y. Hai-qing, "Fault detection algorithm for sensor network based on tendency-similarity," Journal of Huazhong University of Science and Technology (Nature Science Edition), vol. 46, no. 10, pp. 98-104, 2018.

[29] G. Xin, S. Gui-dong, Y. Xiao, and Z. Jing, "Multi-source heterogeneous data fusion recognition based on statistical correlation coefficients between hesitant fuzzy sets," Systems Engineering and Electronics, vol. 40, no. 3, pp. 509-517, 2018.

[30] F. J. Zhao, Z. J. Zhou, C. H. Hu, L. L. Chang, Z. G. Zhou, and G. L. Li, "A new evidential reasoning-based method for online safety assessment of complex systems," IEEE Transactions on Systems, Man, and Cybernetics: Systems, vol. 48, no. 6, pp. 954-966, 2016.

[31] X. Zhou, Y. Hu, Y. Deng, F. T. S. Chan, and A. Ishizaka, “A DEMATEL-based completion method for incomplete pairwise comparison matrix in AHP," Annals of Operations Research, vol. 271, no. 2, pp. 1045-1066, 2018.

[32] L. Yong, L. He, and P. Chuan-jun, "An analytical hierarchy process based quantitative method to evaluate operating condition of thermal power plant," Power System Technology, vol. 39, no. 2, pp. 501-504, 2015. 